\title{
REGISTROS DOS EXAMES COLPOCITOLÓGICOS NAS ESTRATÉGIAS DE SAÚDE DA FAMÍLIA
}

\author{
RECORDS OF THE COLPOCYTOLOGICAL EXAMS IN FAMILY HEALTH \\ STRATEGIES
}

\author{
Laura Izabel Pacheco Pereira Hartmann ${ }^{1}$ \\ Bruna Estevão Araújo² \\ Anna Beatriz Ribeiro Moreira Bazzano ${ }^{3}$ \\ Lisie Souza Castro ${ }^{4}$ \\ Jania Cristiane De Souza Oliveira ${ }^{5}$ \\ Ludiele Souza Castro ${ }^{6}$
}

\begin{abstract}
Resumo: No âmbito da Atenção Primária, o controle da realização dos exames colpocitológicos e seus registros de resultados de forma efetiva são instrumentos fundamentais para adequado controle e manejo clínico do câncer de colo de útero, além de permitir análise do perfil epidemiológico em cada área de abrangência. Com objetivo de analisar os Livros de Registros dos resultados de exames colpocitológicos, 10 Unidades de Saúde da Família foram selecionadas. A pesquisa compreendeu 2.054 registros de exames, destes $84,1 \%$ foram realizados em mulheres com idade entre 25 a 64 anos. Foi observado a ausência de padronização do registro referente aos resultados entre as unidades analisadas, já que foram vistos porcentagens consideráveis de dados sem preenchimento e informação para diferentes aspectos nos exames. Isto é evidenciado quando se observa que em $100 \%$ dos resultados não havia a informação sobre a data da última coleta, em $78,25 \%$ dos registros não informavam a adequabilidade da amostra e $35,64 \%$ dos resultados não definiam quais epitélios estavam presentes no material coletado. Esses dados indicam que o controle dos resultados não está sendo realizado de forma adequada, sendo essencial o treinamento da equipe e padronização dos registros, visando ao aprimoramento.
\end{abstract}

Palavras-chave: Câncer de colo uterino; registros de enfermagem; exame colpocitológico.

Abstract: In the Primary Health Care context, the control of the development of colpocytological exams and their records of results in an effective way are fundamental tools for adequate control and clinical management of cervical cancer, besides allowing analysis of the epidemiological profile in each area of coverage. In order to analyze the Records Books of the results of colpocytological exams, 10 Family Health Units were selected. The survey comprised 2,054 exam records, of which $84.1 \%$ were performed on women aging 25-64 years. The lack of standardization of the record regarding the results among the units analyzed was observed, since there were considerable percentages of unfilled data and information for different aspects of the exams. This is evidenced when it is observed that in $100 \%$ of the results there was no

\footnotetext{
1 Programa de Residência Multidisciplinar em Saúde da Família / Universidade Federal de Mato Grosso. Rondonópolis, MT, Brasil. E-mail: lauraizabelhartmann@gmail.com.

2 Curso de Enfermagem / Universidade Federal de Mato Grosso. Rondonópolis, MT, Brasil. E-mail: brunaraujo159@hotmail.com.

3 Curso de Enfermagem / Universidade Federal de Mato Grosso. Rondonópolis, MT, Brasil. E-mail: annabmoreira@hotmail.com.

4 Curso de Medicina / Universidade Federal de Mato Grosso. Rondonópolis, MT, Brasil. E-mail: lisiescastro@hotmail.com.

${ }^{5}$ Curso de Enfermagem / Programa de Residência Multidisciplinar em Saúde da Família/Universidade Federal de Mato Grosso. Rondonópolis, MT, Brasil. E-mail: jania.ufmt@gmail.com.

6 Curso de Medicina / Programa de Residência Multidisciplinar em Saúde da Família/Universidade Federal de Mato Grosso. Rondonópolis, MT, Brasil. E-mail: tuticastro@hotmail.com.
} 
information about the date of the last collection, $78.25 \%$ of the records did not report the suitability of the sample and $35.64 \%$ of the results did not define which epithelia were present in the sample collected. These data indicate that the control of the results is not being adequately performed, and it is essential to train the staff and standardize the records, aiming to improve the tracking actions and active search of women for cervical cancer prevention.

Keywords: Uterine cervical neoplasm; nursing records; papanicolaou test.

\section{INTRODUÇÃO}

O câncer do colo do útero é uma doença com magnitude considerável em todo o mundo. Em 2012, estimou-se a ocorrência de 527 mil novos casos, caracterizando o quarto tipo de câncer mais comum na população mundial. Cerca de $70 \%$ dos casos diagnosticados de câncer do colo do útero ocorrem em países menos desenvolvidos, porém a taxa de incidência vem diminuindo ao longo das últimas três décadas (INCA, 2015a).

Para os biênios de 2016/2017, no Brasil, foram estimados, segundo o Instituto Nacional do Câncer (INCA), 16.340 casos novos casos de câncer do colo do útero, com um risco estimado de 15,85 casos a cada 100 mil mulheres (INCA, 2015b).

No Estado de Mato Grosso, o INCA analisou taxas brutas de incidência por 100 mil habitantes e sintetizou, aproximadamente, 710 novos casos no estado, sendo, aproximadamente, 430 novos casos só na capital mato-grossense (INCA, 2016a).

O controle do câncer do colo do útero tem como missão a deteç̧ão precoce por meio do rastreamento de lesões precursoras. No Brasil, a prevenção desse tipo de câncer segue a recomendação da realização periódica da coleta do exame colpocitológico.

A periodicidade da realização está na dependência do resultado de exame encontrado em cada caso. Em resultados negativos para malignidade, se esse for 0 primeiro resultado negativo, deve-se realizar novo exame preventivo um ano depois, se já houve um resultado negativo no ano anterior, deverá fazer o próximo exame preventivo após três anos. Porém, se houver positividade de infecção pelo HPV ou lesão de baixo grau, o exame deverá ser repetido após seis meses. No caso de Lesão de alto grau, caberá ao médico a condução do caso, e a realização de outros exames serão necessários, como a colposcopia. Para amostra insatisfatória, o exame deverá ser repetido logo que for possível (INCA, 2015a).

O Papanicolau ou citologia oncótica é um método de análise de melhor benefício para identificar casos que necessitam de encaminhamento para colposcopia e biópsia (ELEUTÉRIOJUNIOR et al., 2004; TUON et al., 2002). A coleta é realizada pelos profissionais enfermeiros e médicos e a análise do material coletado permite a identificação de células sugestivas de pré-invasão e lesões malignas, mediante à coloração multicrômica de lâminas contendo células cervicais esfoliadas. 
Com relação aos resultados obtidos após análise patológica do exame de citologia oncótica, ASCUS é a sigla em inglês para células escamosas atípicas de significância indeterminada, que significa que as anormalidades encontradas nas células cervicais são leves e sua natureza incerta. LSIL, por outro lado, é a sigla em inglês para lesão intraepitelial escamosa de baixo-grau e indica a existência de um maior número de células cervicais anormais definidas, mas de caráter leve. E HSIL ou lesão intraepitelial intraepitelial escamosa de alto-grau indica a existência de células cervicais gravemente anormais, com grande possibilidade de progressão para o câncer do colo de útero (LINS et al., 2014).

No Brasil, é a ferramenta mais utilizada no rastreamento do câncer do colo do útero e, diante de dados, certificou-se que, com a implantação de um rastreamento citológico de qualidade, ampla cobertura da população alvo, tratamento e seguimento das mulheres, é possível reduzir entre 60 a $90 \%$ a incidência do câncer cervical (BRASIL, 2016). A população considerada prioritária para a realização do programa de rastreamento são mulheres com idades entre 25 e 64 anos, tendo em vista a maior ocorrência das lesões de alto grau nessas idades e, se tratadas corretamente, podem não evoluir para o câncer (BRASIL, 2016).

Prevenção, rastreamento e detecção são as melhores estratégias para o câncer. Podemos dividir a prevenção em duas vertentes: a prevenção primária que, além de realizar ações de promoção à saúde, realiza ações focadas em garantir que o câncer não chegue a manifestar; e a prevenção secundária, com o objetivo de detectar e tratar o câncer precocemente, enquanto ainda se encontra em estágio possível de cura (CHAVES, 2010). O acompanhamento terapêutico das mulheres, nos demais níveis de atenção, quando diante de resultado de exame colpocitológico alterado, é também uma das atribuições da Atenção Primária (BRASIL, 2013).

Oportunamente, esse exame é realizado nas consultas ginecológicas, de planejamento familiar, pré-natal e outras (BRASIL, 2013). Após a chegada do resultado de exame da usuária, a Unidade de Saúde da Família (USF) deve ter as informações registradas para controle e acompanhamento. Aos gestores em saúde, cabe salientar que, para o adequado rastreamento de câncer de colo, além da coleta, há necessidade de uma assistência adequada pela equipe interdisciplinar ao interpretar os resultados (CORRÊA; VILLELA, 2008).

O Livro de Registros dos resultados colpocitológicos consiste em um instrumento fundamental para controle, já que todos os dados referentes à identificação e resultado de exame de cada paciente são registrados, permitindo, assim, a todo o momento, monitorar as mulheres da área de abrangência, sendo também importante para o acompanhamento dos números de exames realizados em cada Estratégia de Saúde da Família (ESF) (MINAS GERAIS, 2008). 
Assim, tendo em vista a problemática do câncer de colo de útero para a saúde pública e a necessidade em elaborar estratégias para a melhoria da promoção de saúde, este estudo teve por objetivo analisar os Livros de Registros dos resultados de exames colpocitológicos em USF presentes na cidade de Rondonópolis, no estado de Mato Grosso, a fim de avaliar a qualidade deste instrumento frente ao acompanhamento, busca ativa e tratamento.

\section{MATERIAL E MÉTODO}

Trata-se de um estudo descritivo, analítico, retrospectivo de corte transversal e abordagem quantitativa. $O$ período de coleta de dados ocorreu durante o primeiro semestre de 2017. A pesquisa foi desenvolvida no município de Rondonópolis, localizado na região Sul do Estado de Mato Grosso, com população de, aproximadamente, 195.476 mil habitantes (IBGE, 2010). Esse município possui 40 ESF, divididas em cinco distritos, nomeados de distrito I a distrito $V$. Para a pesquisa, foram sorteadas, aleatoriamente, duas unidades por distrito, totalizando $10 \mathrm{ESF}$.

Foram inclusos registros de exames realizados no ano de 2016 nas unidades sorteadas, no âmbito do Sistema Único de Saúde (SUS), sendo que eles foram analisados no Laboratório Central da Secretaria Municipal de Rondonópolis. E excluídos os que continham somente a informação de coleta realizada, sem registro ou descrição de resultado.

O processo de coleta de dados foi realizado a partir da análise dos Livros de Registros das USF sorteadas. Para esse fim, foi confeccionado um banco de dados em planilha do programa Excel2016 (Microsoft ${ }^{\circledR}$ ), para anotação de todas as informações contidas em cada resultado de exame, englobando: nome da ESF, identificação numeral da lâmina em que foi deposto o material coletado, data da coleta, idade, qualidade da amostra, epitélios identificados, zona de transformação, alterações celulares, microbiologia, malignidade, data da última coleta e observação. Posteriormente, as informações foram analisadas pelo Programa IBM SPSS - Statistics 22.0.

A pesquisa teve aprovação em abril/2017 pelo Comitê de Ética em Pesquisa com Seres Humanos da Universidade Federal de Mato Grosso (UFMT), sob o Parecer oㅜ 369/2017. As Equipes de Estratégia em Saúde da Família que contribuíram para esta pesquisa não foram identificadas, por razões ético-legais.

\section{RESULTADOS}

Um total de 2.159 exames colpocitológicos foram realizados, no período da pesquisa, nas USF participantes, sendo que em 105 exames $(4,87 \%)$ havia somente a informação de coleta e não continham os registros dos resultados, pois eram referentes 
aos exames que não haviam sido retirados pelas pacientes. Assim, o total de resultados analisados foi de 2.054 exames colpocitológicos.

Do total de exames analisados, $84,1 \%(n=1716)$ foram realizados em mulheres entre $25-64$ anos; $15,6 \%(n=319)$, realizados em mulheres entre $18-24$ anos; $0,3 \%$ $(n=6)$, acima de 64 anos; e $0,7 \%(n=13)$ não continham a informação quanto à idade da paciente (Tabela 1). A média de idade foi de 38,2 anos e a mediana de 37 anos.

Tabela 1. Idade das mulheres que realizaram o exame colpocitológico nas Unidades de Saúde da Família, Rondonópolis, MT, 2017.

\begin{tabular}{ccc}
\hline Idade (anos) & $\mathbf{N}^{\circ}$. & $\%$ \\
\hline $18-24$ & 319 & 15,6 \\
$25-34$ & 551 & 27,0 \\
$35-44$ & 519 & 25,4 \\
$45-54$ & 403 & 19,8 \\
$55-64$ & 243 & 11,9 \\
$\geq 65$ & 6 & 0,3 \\
TOTAL & $\mathbf{2 0 4 1}$ & $\mathbf{1 0 0 , 0}$ \\
\hline
\end{tabular}

Nota: Não foi possível ter acesso a idade de 13 mulheres que realizaram o exame colpocitológico.

Quanto à adequabilidade do material (Quadro 1), 78,25\% ( $n=1609)$ dos registros não informavam quanto à classificação satisfatória ou insatisfatória. Descritas como amostras satisfatórias foram $21,6 \%(n=444)$ e como amostra insatisfatória $0,05 \%(n=1)$.

Os dados evidenciaram que dos 2054 resultados, em 35,64\% ( $n=732)$, não havia a descrição dos epitélios representados na amostra. Em 30,6\% ( $n=628)$ dos exames, informavam a presença de epitélio escamoso e glandular; em $29,7 \%$ ( $n=611)$, estavam presentes apenas o epitélio escamoso; em3,5\% $(n=72)$, presentes o epitélio escamoso, glandular e metaplásico; em $0,4 \%(n=9)$, presentes epitélio escamoso e metaplásico; e em $0,1 \%(n=2)$, apenas o epitélio glandular.

Com relação à observação da zona de transformação nas amostras, notou-se a falta dessa informação em 84,42\% $(n=1734)$ dos resultados, em 10,4\% $(n=213)$ dos resultados a zona de transformação estava ausente e em 5,2\% ( $n=107)$ indicavam presença da zona de transformação.

Dentre as alterações celulares benignas reativas ou reparativas, em 19,2\% $(n=396)$ dos resultados das amostras, não foram descritos os achados; em $59,7 \%$ $(n=1.226)$, houve alterações características de inflamação; $0,5 \%(n=1)$, achado com metaplasia escamosa imatura; $0,5 \%(n=1)$, achado com células características de reparação; 5,1\% ( $n=105)$, atrofia com inflamação; $14,4 \%(n=293)$, purulento e outros achados celulares; e em1,65\% ( $n=34)$, achados com outros tipos de alterações. As 
citólises estavam presentes em 6,7 \% $(n=139)$ dos exames descritos nos Livros de Registro das unidades.

No que se refere à identificação microbiológica, podendo conter mais de uma alteração em cada exame, $57 \%(n=1171)$ dos resultados registravam identificação de Lactobacillus sp, 26\% ( $n=538)$ presença de Cocos, $5 \%(n=104)$ Candida sp, $1 \%(n=19)$ Trichomonas vaginalis, $22,1 \%(\mathrm{n}=455)$ Bacilos supracitoplasmáticos (sugestivos de Gardnerella/Mobiluncus). Em 10,22\% ( $n=210)$ dos resultados, não houve descrição no Livro de Registros quanto ao item relacionado identificações microbiológicas.

Foi constatada ausência de informação no Livro de Registro para diferentes aspectos nos exames de citologia oncótica (Quadro 1). Em nenhum Livro, foi registrada a data da última coleta das pacientes.

Quadro 1 - Dados sem informação relacionados aos diferentes aspectos do exame colpocitológico encontrados nos Livros de Registro das Unidades de Saúde da Família, Rondonópolis, 2017.

\begin{tabular}{|l|r|r|}
\hline \multirow{2}{*}{ Dados dos exames colpocitológicos } & \multicolumn{2}{|c|}{ Sem informação } \\
\cline { 2 - 3 } & \multicolumn{1}{|c|}{$N^{\circ}}$. & \\
\hline Adequabilidade do material & 1609 & $78,25 \%$ \\
\hline Epitélio & 732 & $35,64 \%$ \\
\hline Zona de transformação & 1734 & $84,42 \%$ \\
\hline Alterações celulares & 396 & $19,35 \%$ \\
\hline Identificação microbiológica & 210 & $10,22 \%$ \\
\hline Registro da última coleta & 2054 & $100 \%$ \\
\hline
\end{tabular}

Entre os exames que apresentaram algum tipo de lesão, $09(0,4 \%)$ exames obtiveram diagnóstico citológico de lesão intraepitelial de célula escamosa de baixo grau (LSIL) Grau I, e em 01(0,05\%) resultado de exame apresentou lesão intraepitelial de célula escamosa de alto grau (HSIL) Grau II e III. Considerando os resultados dos exames, houve registro de nova solicitação de coleta para, aproximadamente, 130 $(6,32 \%)$ pacientes.

\section{DISCUSSÃO}

Ao considerar o atual cenário nacional de intensificação das ações de prevenção, o alto potencial de cura do câncer de colo do útero, o incremento na oferta de serviços e o tratamento nas diversas fases da doença, todo esse esforço tem seu alcance limitado se as mulheres não retornarem para receber o resultado do exame (VICTOR; MOREIRA; ARAÚJO, 2004). 
Este estudo demonstrou que 105 resultados de exames $(4,87 \%)$ não foram retirados pelas mulheres. Alguns estudos abordam a temática do não retorno das mulheres para receber o resultado do exame, como a falta de recurso para transporte, viagem, esquecimento, trabalho e falta de acolhimento, escuta qualificada e humanização por parte dos funcionários da Unidade de Saúde (GREENWOOD; MACHADO; SAMPAIO, 2006; VICTOR; MOREIRA; ARAÚJO, 2004).

É de extrema importância que se crie uma rotina de verificação das faltas, além de um mecanismo de busca ativa, fazendo uma revisão semanal do Livro de Registro para conhecimento de quais mulheres não compareceram para retirar o resultado do exame colpocitológico. O envio de aerograma, convocação por telefone e realização de visitas domiciliares pelas agentes de saúde são exemplos de mecanismos de busca para essas pacientes (BRASIL, 2013). Aproximadamente, $40 \%$ das mulheres brasileiras nunca se submeteram ao exame de Papanicolau e apenas $7,7 \%$, dessas mulheres, têm acesso a programas governamentais de prevenção e de controle do câncer de colo uterino, para a sua realização (BEGHINI et al., 2006).

A faixa etária prioritária, para detecção do câncer de cérvice uterino, é de 25 a 64 anos de idade, pois é o período que corresponde ao pico de incidência das lesões precursoras que irão progredir para carcinoma invasivo se não detectadas e tratadas em tempo hábil (BRASIL, 2013). No estudo foi possível observar que 84,1\% ( $n=1716)$ dos resultados de exames foram realizados em mulheres nessa faixa etária, e 15,6\% $(n=319)$ dos exames realizados em mulheres abaixo de 25 anos e acima de 65 anos de idade, mostrando congruência com os dados de Reis e colaboradores (2015), que verificou a realização do exame em 70,94\%, em 2009; 67,59\%, em 2010; e 70,48\%, em 2011, na faixa etária preconizada pelo Ministério da Saúde.

No Brasil, no período de 2006 a 2013, os valores observados para a proporção de exames na faixa alvo variaram entre $73,65 \%$ e $78,69 \%$, sendo a média de $76,80 \%$ (COSTA, 2015), revelando similaridade com os resultados da pesquisa em questão. Contudo, cerca de $20 \%$ a $25 \%$ dos exames têm sido realizados fora do grupo etário recomendado e, aproximadamente, metade deles com intervalo de um ano ou menos, quando o recomendado são três anos, o que implica em consumo desnecessário de recursos financeiros públicos. Essa alta frequência de exames fora da faixa etária alvo é verificada, geralmente, em programas de rastreamento oportunístico (ADAB et al., 2004; MADLENSKY et al, 2003), como o do Brasil. Por consequência, há um contingente de mulheres que realizam o exame sem respeitar o adequado intervalo de tempo entre as realizações e outro contingente sem qualquer exame de rastreamento.

Atingir alta cobertura da população definida como alvo é o componente mais importante no âmbito da Atenção Primária, para que se obtenha significativa redução da incidência e da mortalidade por câncer do colo do útero (BRASIL, 2016). Observouse, nos registros coletados neste estudo, a ausência de padronização, referentes aos resultados de exames colpocitológicos, entre as unidades analisadas, resultando em 
falta de informação para diferentes aspectos nos exames, o que acarretou dificuldade à análise do perfil colpocitológico dessas mulheres e impossibilitando seu devido seguimento.

Quanto às alterações cervicais encontradas, as enfermeiras das Unidades não deixaram de relatar no Livro de Registro. Essa informação sugere que, na visão das profissionais, este dado seja o mais importante a ser relatado, dando menor acuidade às outras informações.

Apesar da falta de informação quanto à adequabilidade do material nos exames colpocitológicos, ao avaliar os epitélios presentes nos resultados, foi possível identificar que $32,9 \%$ ( $n=677)$ deles constavam, claramente, que as amostras não apresentavam o epitélio glandular, reafirmando que a inexistência desse epitélio também revela a inexistência da ZT (zona de transformação). A sua ausência limita a visualização e a interpretação da amostra coletada, o que contribui para um elevado índice de resultados falso-negativos, além de associar-se à má qualidade na técnica da coleta dessas células (SILVA et al., 2013).

A metaplasia escamosa consiste em um processo de transformação do epitélio glandular para epitélio escamoso que ocorre, predominantemente, em mulheres mais jovens, tornando-as vulneráveis ao desenvolvimento de lesões intraepiteliais cervicais e ao risco de infecção pelo Papiloma Vírus Humano (HPV). Alguns estudos também apontam a baixa proporção de laudos com a captura do epitélio metaplásico nas amostras coletadas, podendo resultar em laudos falso-negativos que retardam o tratamento direcionado às mulheres com câncer de colo do útero (FERREIRA et al., 2015; SANTOS; MORENO; PEREIRA, 2009). A taxa de falsos negativos pode ser atribuída a três tipos de erro: de amostragem, no rastreamento e na interpretação do esfregaço (RENSHAW, 1997). Mais da metade de todos os resultados falsos negativos é atribuída à amostragem inadequada (GILL, 1997). A baixa incidência de epitélio metaplásico $0,5 \%(n=1)$, registrado no Livro, também se pode associar à baixa representatividade da zona de transformação.

Considerando que apenas 5,2\% ( $n=107)$ dos exames que possuíam células representativas da zona de transformação apresentaram registros em Livro e que as diretrizes nacionais estabelecem que, em todas as amostras, essas células devam estar presentes, há a necessidade de implementar avaliações de monitoramento quanto à efetividade do método de rastreamento de lesões precursoras do câncer do colo uterino. Nesse caso, a qualificação do profissional, mediante as capacitações, pode permear a introdução de medidas diferenciadas para uma prática eficaz (GASPARIN et al., 2016).

Os achados citológicos registrados como citólise, ceratose, binucleação, entre outros, são alterações celulares morfológicas que facilitam a Infecção pelo HPV (GASPARIN et al., 2016). É de grande importância que a presença da citólise seja descrita no laudo do exame colpocitológico e, consequentemente, em Livro de Registro, 
pois, clinicamente, a mulher pode apresentar corrimento abundante de líquido acinzentado, prurido e ardência. Essa secreção apresenta um pH baixo, que gera a proliferação de lactobacilos, predispondo à candidíase de repetição (BRASIL, 2011).

Entre as identificações microbiológicas evidenciadas nos exames, 26\% ( $n=538)$ apresentou presença da microbiota cocobacilar. Em sequência, a contaminação pelo agente microbiológico Gardnerella vaginalis foi identificada em 22,19\% ( $n=455)$ e com a frequência de 5,6\% $(\mathrm{n}=104)$, identificou-se Candida sp.

Estudos mostram elevada frequência de Gardnerella vaginalis, Trichomonas vaginalis, Candida albicans, tradicionalmente associados a vaginoses, bem como do vírus da imunodeficiência adquirida e do HPV, e está relacionada ao aumento no número de parceiros sexuais, bem como à precocidade da sexarca, entre outros fatores (BRASIL, 2013).

Quanto às alterações cervicais, houve predominância da Lesão intraepitelial das células escamosas de baixo grau (LSIL), com 09 (0,4\%) casos, menor que a encontrada em estudo realizado em Brejinho (MA), que apresentou frequência de 1,25\% (SILVA, SANTOS, LIRA FILHO, 2014).

Com relação à Lesão intraepitelial das células escamosa de alto grau (HSIL), houve 1 caso, neste estudo, o qual, no Livro de Registro da Unidade, estava descrito como observação apenas "encaminhamento à colposcopia". Segundo o Ministério da Saúde, as recomendações para conduta frente a esse resultado, são descritos como "colposcopia imediatamente" e em locais em que não esteja garantida a qualidade da citologia ou quando o colposcopista não se sentir seguro quanto à relevância dos achados, a biópsia é aceitável (INCA, 2016b). Essas divergências permitem o questionamento sobre os procedimentos, ou seja, se os demais processos do tratamento estão sendo realizados adequadamente, no tempo recomendado e de forma efetiva.

Como em nenhum Livro foi observado registro da data da última coleta das pacientes, não se sabe como é realizado o controle dos retornos necessários para repetição do exame para cada caso. Este dado, adequadamente descrito em Livro, facilita o estabelecimento da periodicidade de repetição de exames, sendo possível, mediante a revisão do Livro, a verificação das mulheres que requerem um novo exame citopatológico (INCA, 2016b).

É preconizado manter o acompanhamento das pacientes em relação ao exame colpocitológico, com ou sem alterações, na Atenção Básica. Além de ser responsável, também, pelo acompanhamento das pacientes quando contra-referenciado pelo serviço de referência, após diagnóstico ou tratamento, garantindo a humanização do atendimento e adesão ao tratamento dessas mulheres, possibilitando detecção de faltosas (MINAS GERAIS, 2008). 
Importante ressaltar que, para impactar sobre os múltiplos fatores que interferem nas ações de controle dos cânceres do colo do útero, é importante que os profissionais envolvidos estejam empoderados de conhecimento técnico e que a atenção às mulheres esteja pautada em uma equipe multiprofissional e com prática interdisciplinar, envolvendo intervenções na promoção da saúde, na prevenção, no tratamento, na reabilitação. Dentre os principais fatores para garantir sucesso no rastreamento, estão a capacitação dos profissionais, monitoramento do serviço, padronização dos registros e gestão do funcionamento dos sistemas de informação, além de adequado diagnóstico e acompanhamento das mulheres com alterações detectadas (FARIAS, BARBIERI, 2016).

Batista e Gonçalves (2011) alertam que a qualidade do cuidado em saúde ofertado por um sistema está relacionada com a educação permanente de seus profissionais, utilização de protocolos clínicos e definição de linhas de cuidado, formando, assim, profissionais que possam atuar na resolução dos problemas e para a qualificação do cuidado prestado aos sujeitos no sistema de saúde. Diante do exposto, é possível perceber que o processo terapêutico só poderá ser adequado se houver coerência entre as etapas e os registros dos dados. (SANTOS; MORENO; PEREIRA, 2009).

\section{CONCLUSÃO}

A partir da análise dos Livros de Registros presentes nas Unidades de Saúde pesquisadas, foi possível verificar que esse instrumento não está sendo efetivo nos processos de busca ativa e acompanhamento das pacientes. Foi evidenciado que os Livros de Registro não possuem um padrão de preenchimento, os profissionais das Unidades delineiam seu formato, bem como as informações que devem constar. Essa característica ocasionou em falta de informação para diferentes aspectos nos exames, dificultando a análise do perfil colpocitológico das mulheres e impossibilitando o devido acompanhamento das pacientes.

Este estudo indica que problemas gerenciais e técnicos têm dificultado a ampliação e melhoria na qualidade dos serviços ofertados. A necessidade de capacitação técnica dos profissionais que prestam a assistência é fundamental para melhorar o acompanhamento e controle do perfil colpocitológico de mulheres de cada área de abrangência, propiciando, assim, maior suporte advindo da atenção primária, para as mulheres com exames alterados, colaborando para a mudança do perfil epidemiológico do câncer cervical no Brasil.

No presente estudo, houve dificuldade, na coleta de dados, pela falta de padronização do local de registro destes resultados em cada USF pesquisada, e a falta de informação sobre diferentes aspectos nos resultados dos exames dificulta a análise do perfil colpocitológicos de mulheres dessa localidade, o qual poderia contribuir para futuros estudos nessa área. 


\section{AGRADECIMENTOS}

A todas Unidades de Saúde da Família participantes da pesquisa e, em especial, ao Programa de Residência Multidisciplinar em Saúde da Família, da Universidade Federal de Mato Grosso, campus Rondonópolis.

\section{REFERÊNCIAS}

ADAB, $P$. et al. Effectiveness and efficiency of opportunistic cervical cancer screening: comparison with organized screening. Med Care, v. 42, n. 6, p. 600-9, 2004.

BATISTA, C.B.K.; GONÇALVES, J.S.O. Formação dos profissionais de saúde para o SUS: significado e cuidado. Saúde Soc, v. 20, n. 4, p. 884-889, 2011.

BEGHINI, A. B. et al. Adesão das acadêmicas de enfermagem à prevenção do câncer ginecológico: da teoria à prática. Texto contexto - Enferm., v. 15, n. 4, 2006.

BRASIL. Instituto Nacional de Câncer. Programa Nacional de Controle do Câncer de Colo do Útero. Rio de Janeiro: INCA, 2011.

BRASIL. Ministério da Saúde. Protocolos da Atenção Básica: Saúde das Mulheres. Brasília: Instituto Sírio-Libanês de Ensino e Pesquisa, 2016.

BRASIL. Ministério da Saúde. Controle dos cânceres do colo do útero e da mama. 2. ed. Brasília: Secretaria de Atenção à Saúde e Departamento de Atenção Básica, 2013.

CHAVES, A. C. M.; POPPE, M. C. M. Perfil de mulheres submetidas ao exame papanicolau na Unidade Básica de Saúde Moro da Liberdade-Manaus/AMcontribuições da enfermagem para a promoção da saúde. Monografia (Especialização em Saúde da Família) - Universidade Cândido Mendes. Florianópolis, 2010.

CORRÊA, D. A. D.; VILLELA, W. V. O controle do câncer do colo do útero: desafios para implementação de ações programáticas no Amazonas, Brasil. Rev. Bras. Saúde Mater. Infant., v. 8, n. 4, 2008.

COSTA, R. F. Análise da tendência dos indicadores do programa de rastreamento do câncer do colo do útero no Brasil e suas regiões no período de 2006 a 2013. Dissertação (Mestrado Em Ciências da Saúde) - Fundação Pio XII. Hospital de Câncer de Barretos, São Paulo, 2015.

FARIAS, A. C. B.; BARBIERI, A. R. Seguimento do câncer de colo de útero: Estudo da continuidade da assistência à paciente em uma região de saúde. Escola Anna Nery, v. 20 , n. $4,2016$.

FERREIRA, J. E. et al. Perfil da população atendida em um consultório de atendimento integral à saúde da mulher. Cadernos de Graduação Ciências Biológicas e da Saúde, Universidade Tiradentes, v. 3, n. 1, p. 127-140, 2015. 
GASPARIN, V. A. et al. Fatores Associados à Representatividade da Zona de Transformação em exames citopatológicos do colo do útero. Cogitare Enfermagem, v. 21, n. 2, p. 01-09, 2016.

GILL, G. W. Pap smear risk management by process control. Cancer Cytopath., v. 81, n. 4, p. 198-211, 1997.

GREENWOOD S.A; MACHADO M.F; SAMPAIO N.M. Motivos que levam mulheres a não retornarem para receber o resultado de exame Papanicolaou. Revista Latino

Americana de Enfermagem, v. 14, n. 4, p. 503-509, 2006.

IBGE - INSTITUTO BRASILEIRO DE GEOGRAFIA E ESTATÍSTICA. Censo populacional 2010. Disponível em:

<https:cidades.ibge.gov.br/brasil/mt/rondonopolis>. Acesso em: 06 de out. 2017.

INCA. Instituto Nacional de Câncer Jose Alencar Gomes da Silva. Monitoramento das ações de controle do câncer do colo do útero. Informativo detecção precoce: Linha de Cuidado e Rede de Atenção ao Câncer do Colo do Útero. Boletim ano 6, n. 2, maio/ago. 2015a.

INCA. Instituto Nacional de Câncer Jose Alencar Gomes da Silva. Coordenação de Prevenção e Vigilância. Estimativa 2016: Incidência de câncer no Brasil, Rio de Janeiro, 2015b.

INCA. Instituto Nacional de Câncer Jose Alencar Gomes da Silva. Diretrizes brasileiras para o rastreamento do câncer do colo do útero. 2. ed. rev. atual. Rio de Janeiro, 2016a, 114p.

INCA. Instituto Nacional de Câncer Jose Alencar Gomes da Silva. Coordenação de Prevenção e Vigilância. Incidência de Câncer no Brasil, Brasília, 2016b. Disponível em: <http://www.oncoguia.org.br/conteudo/estatistica-para-cancer-de-colodoutero/6717/283/>. Acesso em: 06 de out. 2017.

ELEUTÉRIO JUNIOR, J. et al. Citologia Oncótica, Colposcopia e Histologia no Diagnóstico de Lesões Epiteliais do Colo Uterino. NewsLab, n. 63, p. 126-132, 2004.

LINS, B. et al. Citologia Oncótica: Aplicabilidade e atuação do profissional biomédico na área. In: CONGRESSO DE PESQUISA E EXTENSÄO DA FACULDADE DA SERRA GAÚCHA (FSG), 2, Caxias do Sul, 2014. Anais..., Caxias do Sul, 2014.

MADLENSKY, L. et al. Assessing the evidence for organised cancer screening programmes. Eur J Cancer, v. 39, n. 12, p.1648-53, 2003.

MINAS GERAIS. Secretaria De Estado Da Saúde. Protocolos de Atenção à Saúde da Mulher: Prevenção e controle do câncer do colo do útero. Belo Horizonte, 2008. RENSHAW, A. A. Analysis of error in calculating the false-negative rate in the interpretation of cervicovaginal smears. Cancer Cytopathol., v. 81, n. 5, p. 264-71, 1997.

REIS, A. P. A. et al. Exame citopatológico do colo do útero: diagnóstico situacional de um Centro de Referência. Ciência et Praxis, v. 8, n. 16, 2015. 
SANTOS, M. L.; MORENO M. S.; PEREIRA V. M. Exame Papanicolaou: Qualidade do esfregaço realizado pelos alunos de enfermagem. Revista Brasileira de

Cancerologia, v. 55, n. 1, p. 19-25, 2009.

SILVA, M. G. et al. Determinantes da detecção de atipias celulares no programa de rastreamento do câncer do colo do útero no Rio de Janeiro. Revista Panamericana de Saúde Pública, v. 32, n. 2, p. 107-113, 2013.

SILVA, V.B.; SANTOS, E.P.A.; LIRA FILHO, R. Perfil clínico das mulheres submetidas ao exame papanicolau na USF - brejinho no ano de 2011. Revista Univap, v. 20, n. 35, 2014.

TUON, F.F.B. et al. Avaliação da sensibilidade e especificidade dos exames citopatológico e colposcópico em relação ao exame histológico na identificação de lesões intra-epiteliais cervicais. Rev Assoc Med Bras, v. 48, p. 140-144, 2002.

VICTOR, J. F.; MOREIRA, T. M. M; ARAÚJO, A. R. Exames de prevenção de câncer de colo uterino realizados e não retirados de uma Unidade Básica de Fortaleza Ceará. Acta Paulista de Enfermagem, v. 17, p. 407-411, n. 4, 2004. 